\title{
NOUVELLE
}

\section{Zelda, le maestro du réveil du génome zygotique}

Jeremy Dufourt ${ }^{1}$, Maëlle Bellec ${ }^{1}$, Olivier Messina ${ }^{1}$, Antonio Trullo ${ }^{1}$, Cyril Favard ${ }^{2}$, Ovidiu Radulescu ${ }^{3}$, Mounia Lagha ${ }^{1}$
> Chez de nombreux animaux, bien que la fertilisation aboutisse rapidement à la fusion des génomes maternels et paternels, l'activation du génome zygotique ne débute pas dès les premières heures du développement. En effet, il existe un stock d'ARN et de protéines dans le gamète maternel qui va régir l'embryogenèse précoce. Durant cette première période critique du développement, les cellules totipotentes issues de l'œuf fécondé vont rapidement se différencier pour pouvoir ensuite former les trois feuillets embryonnaires fondamentaux : ectoderme, endoderme, et mésoderme.

\section{La transition materno-zygotique}

Chez tous les métazoaires, le passage graduel du contrôle maternel au contrôle zygotique du développement embryonnaire se déroule lors d'un évènement appelé transition materno-zygotique (MZT), durant laquelle, de manière concomitante à la dégradation des ARN et protéines provenant du gamète maternel, le génome de l'embryon est activé. Cette activation est caractérisée par une vague progressive de transcription, qui coïncide avec le ralentissement des cycles cellulaires dans de nombreuses espèces. Le démarrage de cette vague de transcription ainsi que le nombre de cycles de division qu'elle accompagne varient considérablement d'une espèce à l'autre. Cependant, pour chaque espèce, le déroulement temporel de la MZT est précis et très reproductible d'un embryon à l'autre. Chez les espèces au développement rapide, telles que le nématode (Caenorhabditis elegans), le xénope
(Xenopus laevis), le poisson zèbre (Danio rerio) et la drosophile (Drosophila melanogaster), la MZT s'achève quelques heures seulement après la fécondation, et est suivie du premier évènement de la morphogénèse : la gastrulation. En revanche, chez les espèces à développement plus lent, telles que la souris (Mus musculus) et l'homme (Homo sapiens), la MZT peut durer plusieurs jours [1]. Chez la drosophile, l'embryogénèse commence par 14 cycles nucléaires (nc) rapides au sein d'un syncytium, contrôlés principalement par l'apport en ARN et protéines du gamète maternel. Environ deux heures après la fécondation, le génome zygotique s'active transcriptionnellement et le syncytium se sépare en cellules individuelles (cellularisation) comportant chacune un seul noyau (Figure IA). Le réveil transcriptionnel du génome zygotique nécessite des facteurs de transcription spécifiques, dont la protéine Zelda, qui joue un rôle essentiel dans ce processus [2]. Zelda possède plusieurs caractéristiques des facteurs de transcription dits «pionniers»: 1) sa liaison aux éléments cis-régulateurs avant l'activation des gènes, établissant une pré-compétence pour l'adoption de certains destins cellulaires, et 2) sa capacité à ouvrir la chromatine pour faciliter la liaison de facteurs de transcription classiques. De plus, la distribution homogène de Zelda dans l'embryon permet de potentialiser l'action locale de morphogènes (par exemple, bicoid et dorsal) dans différentes régions de l'embryon [3-5]: la réponse de gènes-cibles à la concentration de morphogène est renforcée par la liaison de Zelda à ces gènes. Cependant, le rôle de ce facteur «pionnier» dans le contrôle temporel de l'activation transcriptionnelle n'a été élucidé que très récemment.

\section{Zelda favorise la coordination} temporelle de la transcription Dès le début de la MZT, le gène snail (sna) est transcrit dans les cellules du futur mésoderme de l'embryon de drosophile. Il code un facteur de transcription qui va permettre la spécification du mésoderme et contrôler la transition épithélio-mésenchymateuse lors de la gastrulation. Dès les premiers cycles nucléaires, la protéine Zelda se lie à deux enhancers (distal et proximal) de sna, qui contrôlent spatialement et temporellement son expression.

Pour tenter de comprendre le rôle de Zelda dans l'activation transcriptionnelle au cours de la MZT, nous avons mesuré les temps d'activation de la transcription, dans les cellules du mésoderme en formation, de plusieurs transgènes ayant une version tronquée de l'enhancer distal de sna (snaE) et possédant un nombre croissant de sites de fixation de la protéine Zelda. Le suivi de l'activation de la transcription dans des embryons vivants est possible grâce à l'ajout, dans une partie non-codante du transgène rapporteur, de séquences de fixation de la protéine du bactériophage MS2 à I'ARN [6]. Lors de la transcrip- 


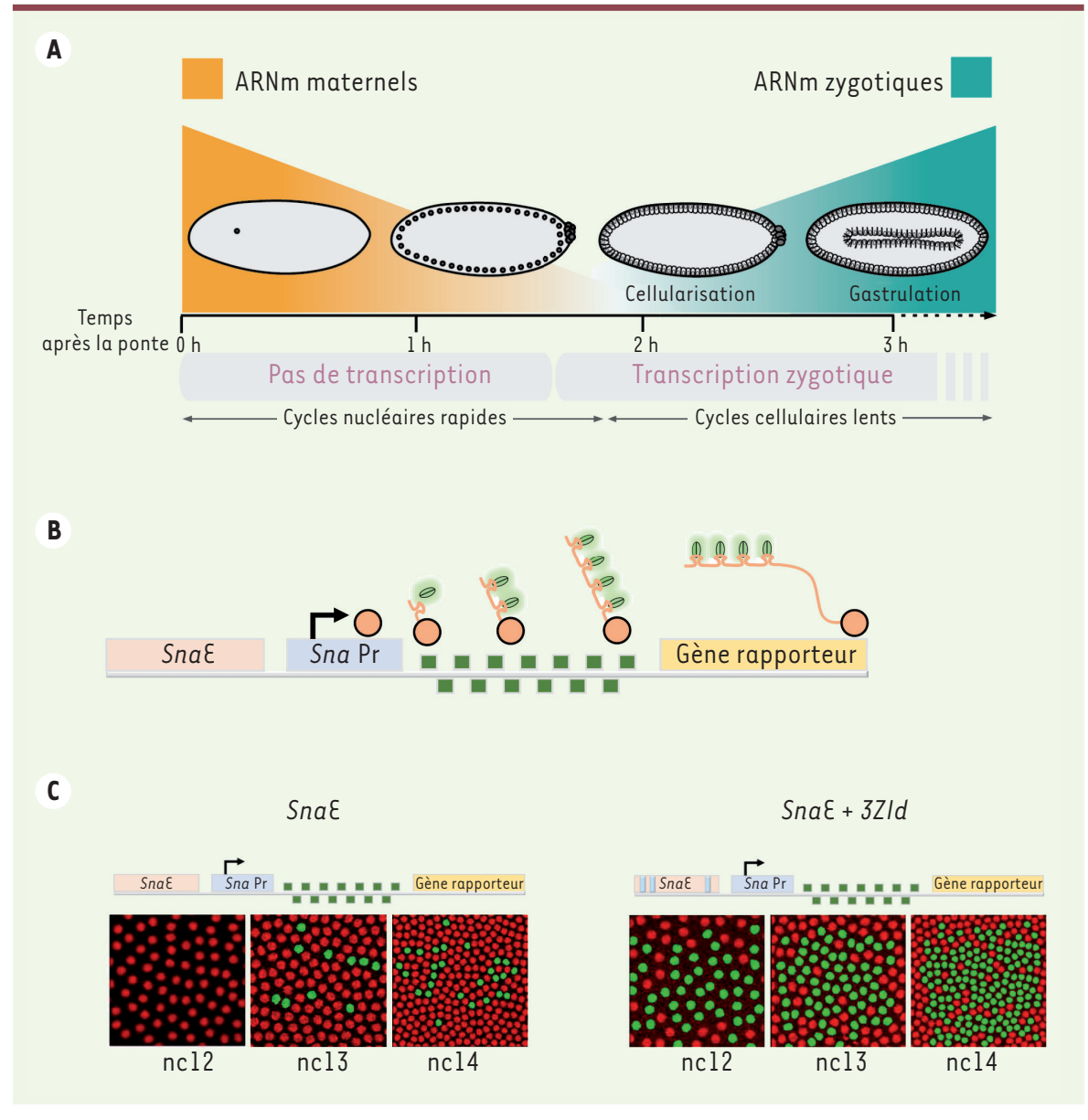

Figure 1. A. Représentation schématique de la transition materno-zygotique (MZT) chez la drosophile. B. Schéma du système MS2/MS2 coat protein (MCP) couplée à la green fluorescent protein (GFP) permettant de visualiser la transcription génique en temps réel. $C$. Représentation schématique des deux transgènes comportant soit la version tronquée de l'enhancer distal de sna (SnaE) ne comportant aucun site de liaison à Zelda, soit cet enhancer avec la présence additionnelle de trois sites de liaison à Zelda $(S n a \varepsilon+3 Z l d)$, et imagerie en temps réel de leur activité de transcription au cours de trois cycles nucléaires (ncl2, ncl3, ncl4). Chaque enhancer (rectangle orange) contrôle le promoteur minimal de sna (SnaPr, rectangle bleu), qui permet la transcription d'un gène rapporteur (rectangle jaune) comportant 24 répétitions du motif de fixation de la protéine MCP (carrés verts) dans sa partie 5'non codante. Les noyaux qui présentent une activité transcriptionnelle sont détectés grâce à la fluorescence de la GFT et colorés en vert. Ils sont beaucoup plus nombreux lorsque l'enhancer distal contient des sites de liaison à Zelda qu'en leur absence.

tion, ces étiquettes forment des « tigesboucles » avec I'ARNm, auxquelles va rapidement se lier une protéine de fusion fluorescente (MS2 coat protein couplée à la green fluorescent protein), ce qui permet de visualiser ces nouveaux transcrits (Figure 1B). Nous avons montré que la protéine Zelda accélérait la coordination de l'activation de la transcription (synchronie) de sna au sein du mésoderme en formation (Figure 1C).

\section{Zelda masque la mémoire transcriptionnelle}

La possibilité de suivre l'activation de la transcription dans des embryons vivants permet aussi de déterminer l'effet de Zelda sur l'activité transcriptionnelle au cours de multiples cycles nucléaires. Nous avons ainsi récemment documenté l'existence d'une mémoire mitotique transcriptionnelle, en montrant que le statut transcriptionnel des noyaux mères lors d'un cycle nucléaire donné influençait l'activation de la transcription, au cycle suivant, dans les noyaux qui en dérivent [7] (Figure 2A).

Les cellules vont se diviser de nombreuses fois au cours du développement, et la régulation génique des cellules mères doit être transmise aux cellules filles afin de maintenir l'identité tissulaire. Cependant, à chaque division cellulaire (mitose), la transcription s'arrête, les chromosomes se condensent, et la plupart des facteurs de transcription se dissocient de la chromatine. Afin de transmettre l'information d'identité cellulaire, certains mécanismes garantissent la réactivation post-mitotique de la transcription de certains gènes d'identité dans les cellules filles. Ce processus est appelé «bookmarking » mitotique en référence aux facteurs qui restent associés à la chromatine durant la mitose et servent de «marque-pages » [8]. Les facteurs de transcription «pionniers », outre leur propriété de se lier aux nucléosomes, restent généralement associés aux chromosomes mitotiques. La rétention mitotique de ces facteurs de transcription en fait des candidats idéaux pour la transmission de l'identité cellulaire au cours des divisions mitotiques.

Sous l'effet de l'activation de ses enhancers, l'expression de sna est très rapide, ce qui empêche d'analyser le rôle de Zelda dans le tempo d'activation de sna. Nous avons donc utilisé la version tronquée de l'enhancer distal de sna (snaE) qui ne contient plus de sites de fixation de la protéine Zelda, et lui avons ajouté, ou pas, des sites de fixation de Zelda. L'activation de ce transgène est stochastique, mais toujours restreinte aux noyaux du mésoderme en formation. Ce dispositif expérimental permet de suivre les temps d'activa- 
A

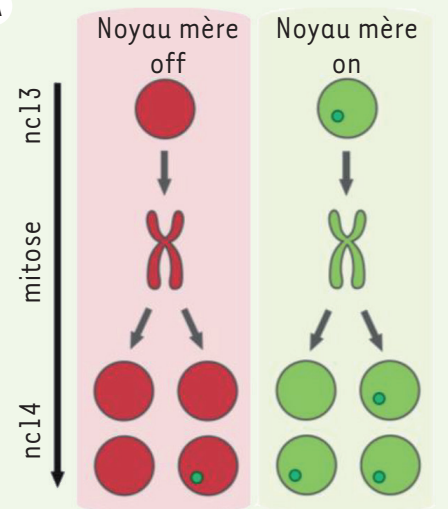

C

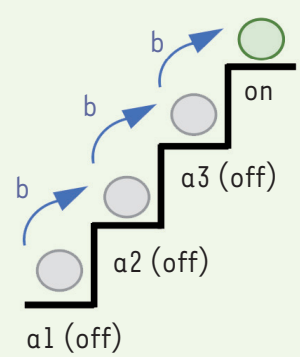

B

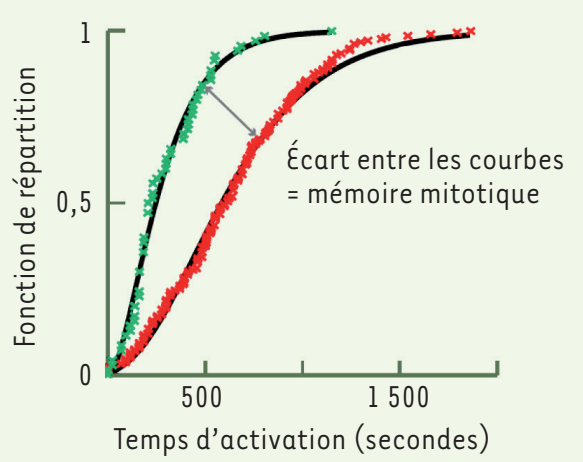

D GFP-Zelda

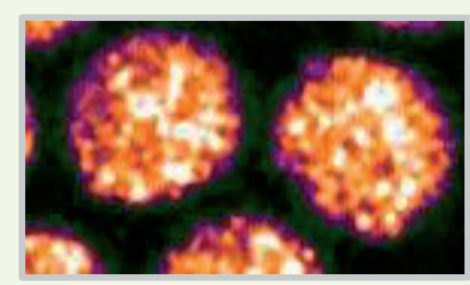

Figure 2. A. Schéma représentant la mémoire mitotique entre deux cycles nucléaires consécutifs (nc 13 et ncl4) de l'embryon de drosophile. Les noyaux issus de noyaux mères dans lesquelles la transcription génique est active (on, en vert) s'activent en moyenne plus rapidement que ceux issus de noyaux mères dans lesquelles la transcription génique est inactive (off, en rouge), au cycle nucléaire suivant (ncl4). B. Fonction de répartition des temps d'activation au cycle nucléaire 14. Les valeurs des temps d'activation transcriptionnelle des noyaux issus de noyaux mères «actifs » sont représentées en vert, et celles des noyaux issus de noyaux mères «inactifs» sont représentées en rouge. Les paramètres du modèle mathématique sont estimés à partir de courbes ajustées à ces valeurs expérimentales (courbes noires). C. Représentation schématique des états épigénétiques discrets nécessaires pour atteindre l'activation de la transcrip-

tion (état $\mathrm{ON}$ ). La durée de chaque transition d'un état à l'autre est fournie par le paramètre noté « $b$ ». D. Image de microscopie confocale de la protéine Zelda étiquetée avec une GFP (par la technique CRISPR/Cas9) montrant la présence de « microenvironnements nucléaires » dans lesquels la protéine s'accumule. $\varepsilon$. Schéma représentant le rôle de Zelda dans l'activation synchrone de la transcription de multiples gènes zygotiques. ZLD : Zelda; GFP : green fluorescent protein.

tion transcriptionnelle des noyaux filles provenant de noyaux mères transcriptionnellement «actifs » ou « inactifs » (Figure 2B): ces temps d'activation reflètent la mémoire transcriptionnelle à travers la mitose [7]. Nous avons pu ainsi montrer que Zelda accélérait l'activation transcriptionnelle des deux sous-populations de noyaux filles. Par une accélération générale de la transcription, Zelda tend ainsi à diminuer le biais de mémoire mitotique. Cependant ce biais de mémoire est récupéré lorsqu'on diminue l'expression de ce facteur dans l'embryon, ce qui suggère l'implication d'autres facteurs dans le processus de mémoire mitotique.

Pour affiner notre compréhension du rôle biologique de Zelda dans l'activation du génome zygotique, nous avons formalisé nos données en développant un modèle mathématique, qui permet de mieux appréhender le nombre et la durée des étapes nécessaires à l'activation de la transcription. Dans ce modèle, l'activation de la transcription est atteinte après avoir parcouru une série de transitions aléatoires entre états épigénétiques discrets (Figure 2C). Le modèle prédit que le type de distribution du temps d'activation (loi exponentielle, loi gamma, etc.) dépend du nombre d'états discrets. L'analyse des résultats expérimentaux en utilisant ce modèle indique qu'en moyenne, les noyaux provenant de noyaux mères, dans lesquels la transcription est active, doivent parcourir un nombre plus petit de transitions jusqu'à l'activation, et suggère 
que Zelda agit principalement en diminuant la durée (et non le nombre) des étapes de pré-initiation de la transcription, révélant ainsi son rôle accélérateur dans l'embryon précoce de drosophile.

\section{Zelda se fixe transitoirement} à la chromatine

Compte tenu des caractéristiques des facteurs pionniers, nous nous attendions à ce que Zelda joue un rôle dans la conservation de la mémoire transcriptionnelle durant la mitose. Cependant, nos données génétiques et notre modélisation indiquent que Zelda n'est pas le support de cette mémoire. Nous avons analysé la localisation de Zelda dans des noyaux d'embryons vivants et avons montré que Zelda quitte le noyau à chaque mitose, mais y retourne rapidement en fin d'anaphase. Afin de préciser les propriétés dynamiques de cet activateur du génome, nous avons eu recours à des expériences d'imagerie quantitative (fluorescence recovery after photobleaching et fluorescence correlation spectroscopy) sur embryon vivant. Nous avons montré que ce facteur de transcription «pionnier» ne se liait à la chromatine que très transitoirement, avec un temps de résidence estimé de l'ordre de quelques secondes. Cependant, ce faible temps de résidence de la protéine Zelda sur la chromatine pourrait être compensé par une augmentation locale de sa concentration. En effet, nous avons montré que Zelda s'accumu- lait au sein de «microenvironnements » nucléaires (hubs) (Figure 2D), ce qui a aussi été rapporté par une autre équipe en utilisant une technique de microscopie à haute résolution (lattice light sheet) permettant de suivre des particules uniques [4]. L'accumulation de Zelda dans ces «microenvironnements » nucléaires pourrait favoriser une coopérativité entre plusieurs facteurs de transcription [4] ou le rapprochement de plusieurs segments d'ADN cibles (enhancers) pour favoriser l'activation transcriptionnelle des gènes zygotiques (Figure 2E).

Ces travaux de recherche [9] ouvrent de nouvelles pistes pour mieux comprendre le rôle des facteurs de transcription «pionniers» (e.g. Oct4, Pou5f3, Sox2), l'activation des gènes et l'organisation nucléaire durant la période d'activation du génome zygotique chez les vertébrés. Les gènes codant des facteurs de transcription «pionniers», tels que ceux de la famille Forkhead box (Fox), sont impliqués dans de nombreuses maladies génétiques complexes telles que les cancers, la maladie de Parkinson ou les troubles du spectre autistique [10]. De ce fait, la compréhension de l'activité spatio-temporelle de ces facteurs de transcription «pionniers» permettrait de progresser dans la physiopathologie de ces maladies dans le but d'identifier de nouvelles stratégies thérapeutiques. $\diamond$

Zelda, maestro of the zygotic genome awakening

\section{REMERCIEMENTS}

Nous remercions Matthieu Dejean pour sa lecture critique du manuscrit. Ces travaux ont été soutenus par l'ERC SyncDev et une subvention HFSP-CDA.

\section{LIENS D'INTÉRÊT}

Les auteurs déclarent n'avoir aucun lien d'intérêt concernant les données publiées dans cet article.

\section{RÉFÉRENCES}

1. Tadros W, Lipshitz HD. The maternal-to-zygotic transition: a play in two acts. Development 2009; 136 : 3033-42.

2. Hamm DC, Harrison MM. Regulatory principles governing the maternal-to-zygotic transition: insights from Drosophila melanogaster. Open Biol 2018 ; 8 : 180183.

3. Yamada S, Whitney PH, Huang SK, et al. The drosophila pioneer factor Zelda modulates the nuclear microenvironment of a dorsal target enhancer to potentiate transcriptional output. Curr Biol 2019 ; 29: 1387-93.

4. Mir M, Stadler MR, Ortiz SA, et al. Dynamic multifactor hubs interact transiently with sites of active transcription in Drosophila embryos. Elife 2018 ; 7 (10.7554/elife.40497).

5. Mir M, Reime A, Haines JE, et al. Dense Bicoid hubs accentuate binding along the morphogen gradient. Genes Dev 2017 ; 31 : 1784-94.

6. Bertrand $\varepsilon$, Chartrand P, Schaefer M, et al. Localization of ASH1 mRNA particles in living yeast. Mol Cell $1998 ; 2: 437-45$.

7. Ferraro T, Esposito $\varepsilon$, Mancini L, et al. Transcriptional memory in the drosophila embryo. Curr Biol 2016; 26 : 212-8.

8. Bellec M, Radulescu 0, Lagha M. Remembering the past: mitotic bookmarking in a developing embryo. Curr Opin Syst Biol 2018 ; 11 : 41-9.

9. Dufourt J, Trullo A, Hunter J, et al. Temporal control of gene expression by the pioneer factor Zelda through transient interactions in hubs. Nat Commun 2018 ; 9 : 5194.

10. Golson ML, Kaestner KH. Fox transcription factors: from development to disease. Development 2016 ; $143: 4558-70$.

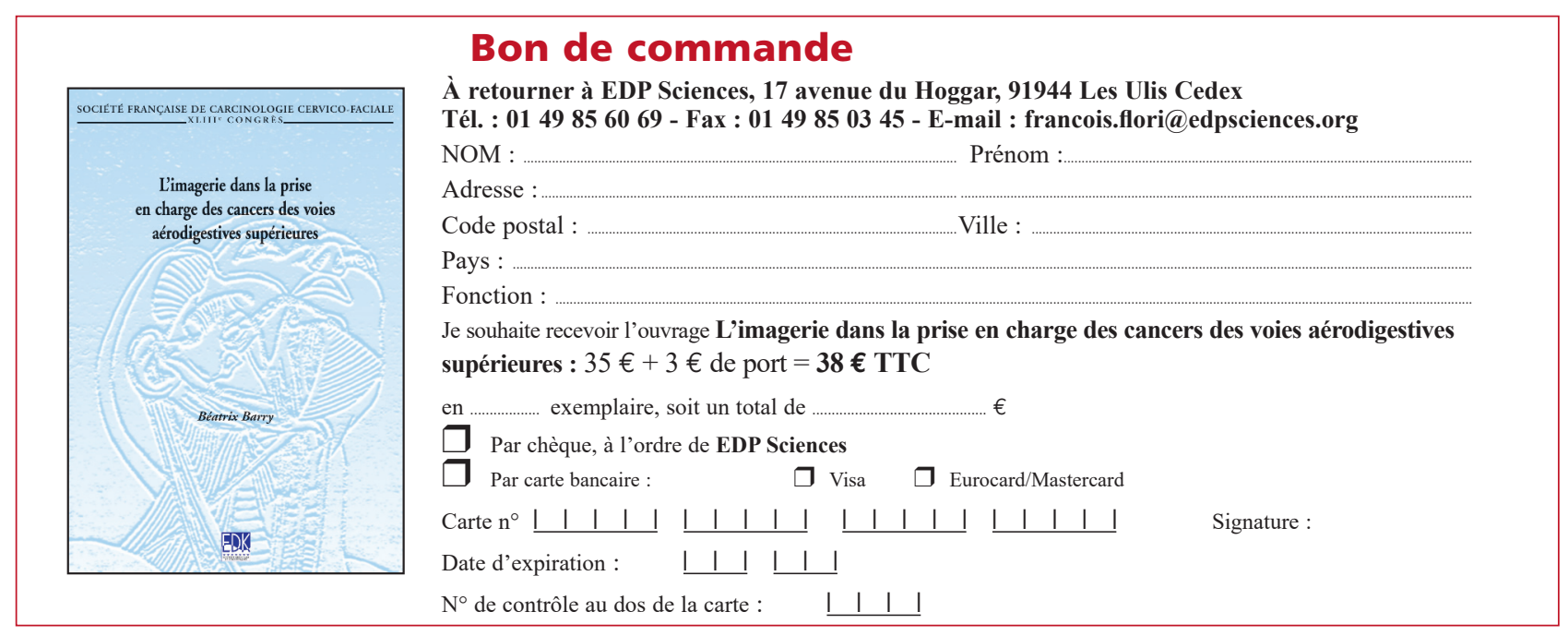

